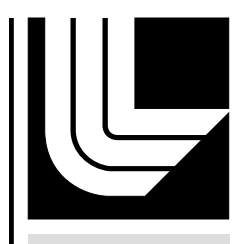

LAWRENCE LIVERM ORE N A TIO NAL LABORATORY

Simulating the Rayleigh-Taylor instability with the Ising model

J. R. Ball, J. B. Elliott

September 1, 2011 
This document was prepared as an account of work sponsored by an agency of the United States government. Neither the United States government nor Lawrence Livermore National Security, LLC, nor any of their employees makes any warranty, expressed or implied, or assumes any legal liability or responsibility for the accuracy, completeness, or usefulness of any information, apparatus, product, or process disclosed, or represents that its use would not infringe privately owned rights. Reference herein to any specific commercial product, process, or service by trade name, trademark, manufacturer, or otherwise does not necessarily constitute or imply its endorsement, recommendation, or favoring by the United States government or Lawrence Livermore National Security, LLC. The views and opinions of authors expressed herein do not necessarily state or reflect those of the United States government or Lawrence Livermore National Security, LLC, and shall not be used for advertising or product endorsement purposes.

This work performed under the auspices of the U.S. Department of Energy by Lawrence Livermore National Laboratory under Contract DE-AC52-07NA27344. 


\title{
LLNL-TR-497289: Simulating the Rayleigh-Taylor instability with the Ising model
}

\author{
Justin Ball and James Elliott \\ September 2, 2011
}

\section{Description of Model}

The model consists of a two dimensional Cartesian Ising lattice of flippable spins, $\hat{s} \in\{-1,+1\}$. Spin exchange probabilities were determined according to the Metropolis algorithm, with a single time step defined as follows:

1. Propose a new configuration via random change to the initial configuration

2. Calculate the energy difference, $\Delta E$, between the two configurations

3. Generate a random number $0 \leq r \leq 1$

4. If $r \leq\left\{\begin{array}{ll}1 & \Delta E<0 \\ e^{\frac{-\Delta E}{k_{B} T}} & \text { else }\end{array}\right.$, then keep the new configuration, otherwise keep the old configuration

Kawasaki dynamics provides the method for proposing new configurations. A single nearest neighbor pair is selected, uniformly at random, from the initial configuration and swapped to produce the proposed configuration. This method has the effect of conserving the number of each type of spin which, in the interpretation of our system, represents conservation of mass.

The energy difference, $\Delta E \equiv \Delta E_{\text {bond }}+E_{\text {grav }}$, is calculated from the effect of the traditional nearest neighbor bond force (leading to $\Delta E_{\text {bond }}$ ) and a 
LLNL-TR-497289: Simulating the Rayleigh-Taylor instability with the Ising model

gravitational force (leading to $\Delta E_{\text {bond }}$ ). To determine the effects of the bond force, a spin interaction energy, $J$, is prescribed and a configuration is defined to have a total bond energy given by

$$
E_{\text {bond }} \equiv \frac{-J}{2} \sum_{\hat{s}_{i}=\text { all spins } \hat{s}_{j}=\text { nearest neighbors of } \hat{s}_{i}} \hat{s}_{i} \cdot \hat{s}_{j} .
$$

Given the constraints of Kawasaki dynamics, the bond energy difference, $\Delta E_{\text {bond }}$, between an initial state and a proposed state, generated by swapping a single pair of spins $\hat{s}_{i}$ and $\hat{s}_{j}$ in the initial state, can be calculated locally. It is given by

$$
\begin{aligned}
\Delta E_{\text {bond }} & =-J\left[\left(\sum_{\hat{s}_{k}=\text { nearest neighbors of } \hat{s}_{i}} \hat{s}_{i} \cdot \hat{s}_{k}+\sum_{\hat{s}_{k}=\text { nearest neighbors of } \hat{s}_{j}} \hat{s}_{j} \cdot \hat{s}_{k}\right)_{\text {proposed }}\right. \\
& \left.+\left(\sum_{\hat{s}_{k}=\text { nearest neighbors of } \hat{s}_{i}} \hat{s}_{i} \cdot \hat{s}_{k}+\sum_{\hat{s}_{k}=\text { nearest neighbors of } \hat{s}_{j}} \hat{s}_{j} \cdot \hat{s}_{k}\right)_{\text {initial }}\right] .
\end{aligned}
$$

The total gravitational energy of a configuration, after prescribing spin masses $m_{+1}$ and $m_{-1}$ to +1 and -1 spins respectively, a gravitational constant $g$, and a lattice spacing $\Delta h$, is

$$
E_{\text {grav }} \equiv g \sum_{\hat{s}_{i}=\text { all spins }} m_{i} y_{i} \Delta h,
$$

where $y_{i}$ and $m_{i}$ are the row number and mass of spin $\hat{s}_{i}$. The gravitational energy difference between an initial state and a proposed state, generated by swapping spins $\hat{s}_{i}$ and $\hat{s}_{j}$, in the initial state is

$$
\Delta E_{\text {grav }}=\left(m_{i}-m_{j}\right) g\left(h_{i}-h_{j}\right) .
$$

Since, spins can only swap with their neighbors the definition

$$
E_{\text {drop }} \equiv\left(m_{+1}-m_{-1}\right) g \Delta h
$$


LLNL-TR-497289: Simulating the Rayleigh-Taylor instability with the Ising model

is useful. The gravitational energy difference for any swap of adjacent similar spins (both +1 or both -1 ) is zero because the initial and proposed configurations are indistinguishable. However, the gravitational energy difference for any swap of adjacent dissimilar spins $\hat{s}_{+1}$ and $\hat{s}_{-1}$, originally at heights $y_{+1}$ and $y_{-1}$, is

$$
\Delta E_{\text {grav }}= \begin{cases}E_{\text {drop }} & y_{+1}>y_{-1} \\ -E_{\text {drop }} & y_{+1}<y_{-1} \\ 0 & y_{+1}=y_{-1}\end{cases}
$$

The input parameters to the simulation are the gravitational strength, $E_{\text {drop }}$ (in units of the spin interaction energy, $J$ ), the temperature, $k_{B} T$ (in units of $J$ ), and the lattice dimensions $n_{x}$ and $n_{y}$ (in units of the lattice spacing, $\Delta h$ ). Periodic boundary conditions were used on the boundaries normal to the $\hat{x}$ direction. Finite, fixed boundary conditions were used on the boundaries normal to the $\hat{y}$ direction. No edge existed in the $+\hat{y}$ direction from the top row of spins or in the $-\hat{y}$ direction from the bottom row of spins. With these boundary conditions the total number of nearest neighbor pairs is $N_{\text {bonds }}=2 n_{x} n_{y}-n_{x}$. The code was written is $\mathrm{C}++$ and run on a single processor.

\section{Model Benchmarking}

In order to benchmark our model, we tested the effects of gravitational and bond forces independently. Both could be removed easily and analyzed theoretically, but isolated verification still gives confidence that they will function as intended in concert.

\subsection{Gravitational Forces}

To benchmark the effect of gravitational forces, bond forces were removed. This was done by removing its contribution when calculating the energy difference of a proposed swap. Normally, the probability of a spin pair flipping depends on the six spins surrounding the pair, but without bond forces, it depends only on the two spins involved. The lack of significant collective effects makes the following assumption approximately true: the probability of 
LLNL-TR-497289: Simulating the Rayleigh-Taylor instability with the Ising model

a given lattice site being +1 is independent of the probability of its neighbors being +1 . Usually this is a bad assumption because bond forces cause like spins to clump, so having +1 neighbors makes it more likely for the given site to be +1 itself. This assumption allows us to create a mathematical method to deterministically approximate the statistical evolution of any arbitrary simulation.

We begin with initial conditions, a finite $n_{x} \times n_{y}$ lattice with a distribution of +1 and -1 spins, and boundary conditions (in this case periodic in $\hat{x}$ and fixed in $\hat{y}$ ). We take our initial conditions and translate them into a function $S(0, x, y) \rightarrow[0,1]$ over $x \in\left[0, n_{x}\right), y \in\left[0, n_{y}\right)$ where $S(0, x, y)$ maps to 0 at the locations of -1 spins and 1 at the locations of +1 spins. The probability of a lattice site being +1 is the sum of the probability that it was +1 last time step times the probability that it didn't swap with a -1 and the probability that it was -1 last time step times the probability that it did swap with $a+1$. This can be written, in our previously established notation, as the recurrence relation

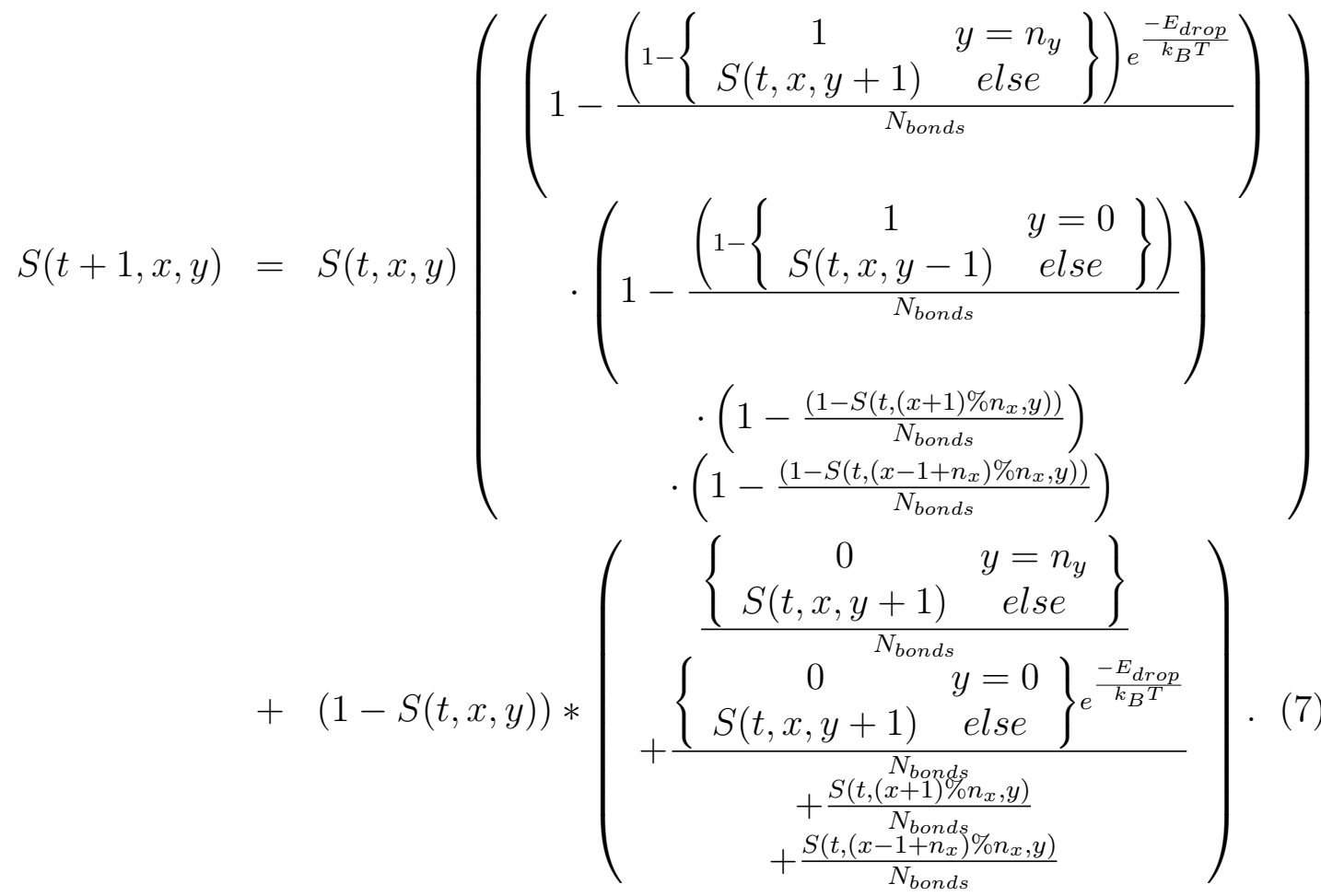

Equation 7 was solved iteratively from the initial conditions appearing 
LLNL-TR-497289: Simulating the Rayleigh-Taylor instability with the Ising model

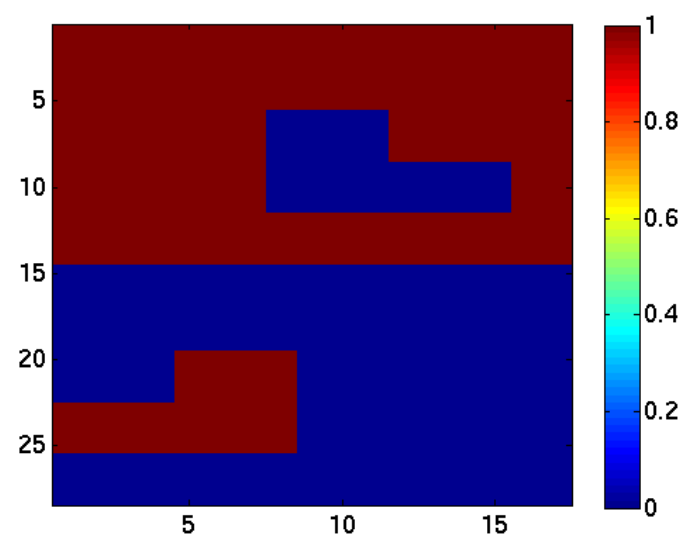

Figure 1: Initial conditions used for gravitational force benchmarking

in figure 1 to give the probability distribution given in figure 2 at $t=5000$ considerations. This theory was compared with the distribution in figure 3 , generated by averaging over 100,000 simulation runs. The mean absolute difference was $0.5 \%$ and the maximum absolute difference was $2.5 \%$ across all lattice sites.

\subsection{Bond Forces}

To benchmark the effect of bond forces, the effects of gravity we removed. We started with a $100 \times 400$ lattice $\left(n_{x}=100, n_{y}=400\right)$ with a flat interface with a normal in the $\hat{y}$ direction. The lattice was allowed to reach equilibrium at various temperatures between 0 and $4 J$, after which the average bond energy per spin was calculated. A theoretical prediction was determined through modifying the solution for the average bond energy in the grand canonical ensemble.

In the grand canonical ensemble the lowest energy state is a homogeneous lattice of spins. Our system uses the canonical ensemble which, with heterogeneous initial conditions, necessitates a spin interface in the lowest energy state. If the lattice size is infinite the two cases, canonical and grand canonical, become equivalent because the number of interface bonds is $O\left(n_{x}\right)$ and the total number of bonds is $O\left(n_{x} n_{y}\right)$. For a finite lattice, the difference between the lowest energy states of the two cases can be calculated from the 
LLNL-TR-497289: Simulating the Rayleigh-Taylor instability with the Ising model

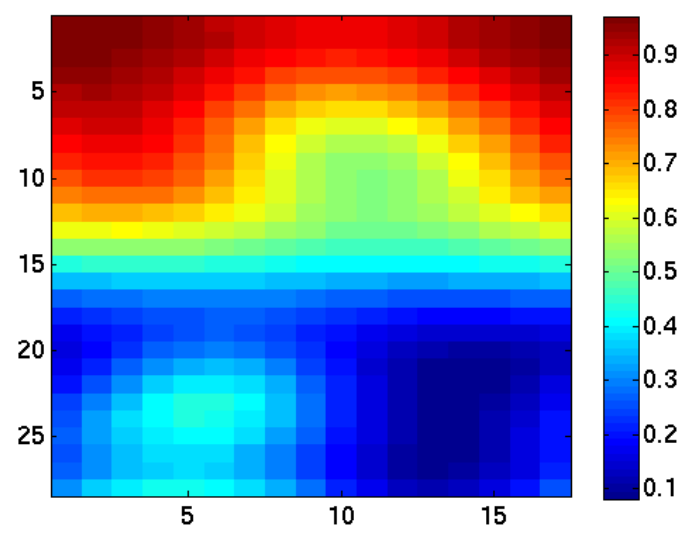

Figure 2: Theoretical probability distribution at $\mathrm{t}=5000$ time steps

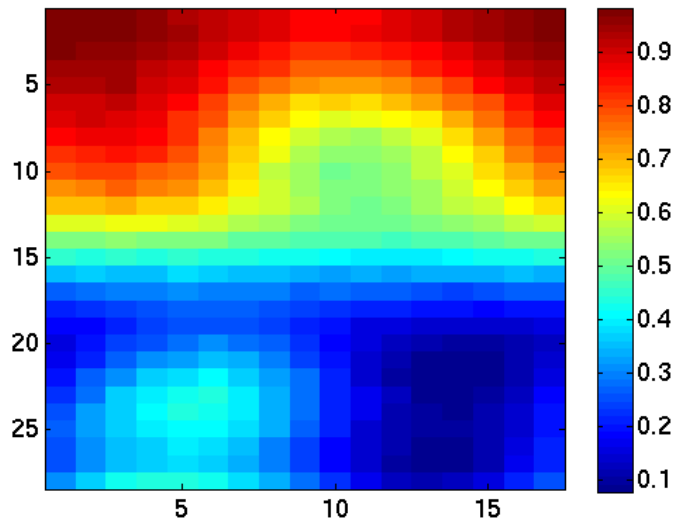

Figure 3: Simulation probability distribution at $\mathrm{t}=5000$ time steps, averaged over 100,000 runs 
LLNL-TR-497289: Simulating the Rayleigh-Taylor instability with the Ising model

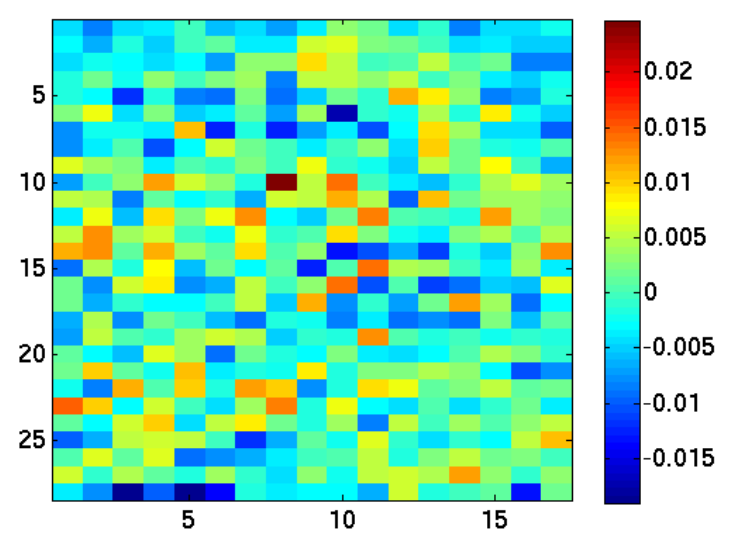

Figure 4: Simulation results subtracted from theoretical prediction of probability distribution at $\mathrm{t}=5000$ time steps

ratio of interface to total bonds to be

$$
\Delta\left\langle E_{\text {bond }}\right\rangle_{\text {finite }}=\frac{3 J}{n_{y}} .
$$

Now, with the adjustment to the finite canonical result, it becomes

$\left\langle E_{\text {bond }}\right\rangle=\frac{3 J}{n_{y}}-\operatorname{coth}\left(\frac{2}{k_{B} T}\right)\left(1-\left(1-2 \tanh ^{2}\left(\frac{2}{k_{B} T}\right)\right) \frac{2}{\pi} K\left(\frac{2 \sinh \left(\frac{2}{k_{B} T}\right)}{\cosh ^{2}\left(\frac{2}{k_{B} T}\right)}\right)\right) J$

where $K(k)=F\left(\frac{\pi}{2} \mid k^{2}\right)=\int_{0}^{\frac{\pi}{2}} \frac{d \theta}{\sqrt{1-k^{2} \sin ^{2}(\theta)}}$ is the complete elliptic integral of the $1^{\text {st }}$ kind. This theory is compared against the results of simulations in figure 5. The mean absolute difference is $6 \%$ and the maximum absolute difference is $17 \%$.

\subsection{Critical Temperature Estimation}

In order to estimate the critical temperature, $k_{B} T_{c}$, of the system we can apply equation 10 for free energy, $F$, to the situation of initially segregated 
LLNL-TR-497289: Simulating the Rayleigh-Taylor instability with the Ising model

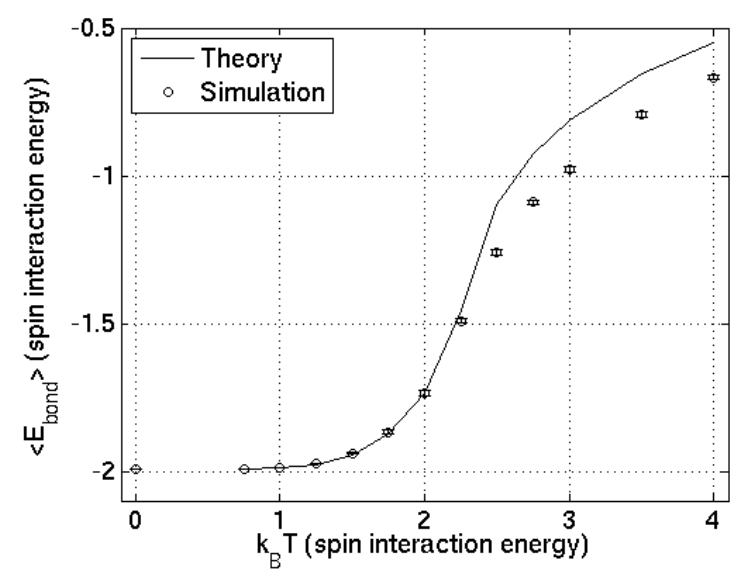

Figure 5: Average bond energy per spin for simulation and theory

species without the influence of gravity. We arbitrarily chose the top half of the lattice to be +1 spins and the bottom half to be -1 spins. At equilibrium the two species have mixed at the interface to a degree dependent on the lattice temperature, $k_{B} T$. By estimating the energy, $E$, and the entropy, $S$, of the mixing region as functions of $k_{B} T$, we can minimize the free energy and arrive at an estimation of the critical temperature.

$$
F=E-T S
$$

\subsubsection{Simplifying Assumptions}

The following assumptions were made to approximate Onsager's result for the critical temperature of the two dimensional infinite Cartesian Ising lattice:

1. The vertical spin composition profile is an error function

2. The horizontal spin distribution is uniformly random

Simulations show the probability of a given lattice site being +1 as a function of height of the mixing region is fit by an error function reasonably well (see figure 6 , where $\omega_{1}, \omega_{2}, \omega_{3}$, and $l$ are fit parameters). Taking the derivative of the error function fit, we can get a Gaussian distribution with 
LLNL-TR-497289: Simulating the Rayleigh-Taylor instability with the Ising model

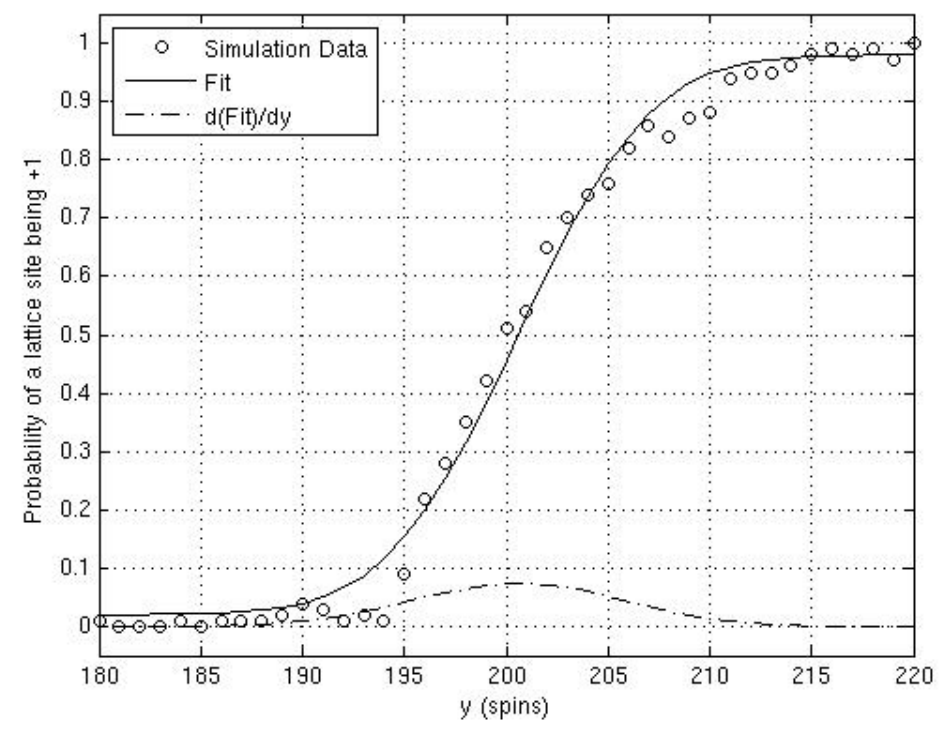

Figure 6: Error function fit, Fit $=\omega_{1}\left(\operatorname{erf}\left(\frac{2 \sqrt{\ln (2)}}{l}\left(y-\omega_{2}\right)\right)\right)+\omega_{3}$, to simulation data, $n_{x}=100, n_{y}=400, E_{\text {drop }}=0$, and $k_{B} T=1.75 \mathrm{~J}$

a full-width at half maximum (FWHM), $l$, characteristic of the width of the mixing region.

In addition to assuming an error function profile, we must also assume a relationship between bond energy and density. For example, near the top of the lattice there are very few -1 spins, so they have almost entirely dissimilar neighbors. But near the interface there is a large population of -1 spins, which will often be adjacent, reducing the energy, $E$. For this approximation we will assume that, at a given height, the probability of a spin occupying a lattice site is independent of the occupants of neighboring sites (thermal effects dominate bond effects).

\subsubsection{Energy Calculation}

Using these assumptions we can calculate the energy of the interface. At a given height $y$ above the interface, if $f(y)$ is the chance of being +1 , there are $n_{x}(1-f(y))-1$ spins. Each spin has 4 neighbors each with a $f(y)$ chance of 
LLNL-TR-497289: Simulating the Rayleigh-Taylor instability with the Ising model

being +1 . This argument is symmetric about the initial interface meaning the total bond energy is just twice the bond energy above the initial interface. If we let $f(y)=\operatorname{erf}\left(\frac{2 y \sqrt{\ln (2)}}{l}\right)$ and let $n_{y}=\infty$, we arrive at

$$
\begin{aligned}
\frac{E}{2} & =\int_{0}^{\infty} 4 n_{x} \operatorname{erf}\left(\frac{2 y \sqrt{\ln (2)}}{l}\right)\left(1-\operatorname{erf}\left(\frac{2 y \sqrt{\ln (2)}}{l}\right)\right) J d y \\
E & =\frac{4 n_{x} l}{\sqrt{\pi \ln (2)}}(\sqrt{2}-1) J
\end{aligned}
$$

\subsubsection{Entropy Calculation}

Entropy is defined to be the $k_{B}$ times the natural log of the number of states possible which satisfy the constraints of the system. In this calculation the only constraint is the area $(A)$ in which mixing is permitted to occur. The mixing of two species in an area has associated entropy given by

$$
S=k_{B} \cdot \ln \left(\left(\begin{array}{c}
2 A \\
A
\end{array}\right)\right)=2 A k_{B} \ln (2) .
$$

For the assumed profile, $A=\int_{0}^{\infty} \operatorname{erf}\left(\frac{2 y \sqrt{\ln (2)}}{l}\right) d y=\frac{n_{x} l}{2 \sqrt{\pi \ln (2)}}$, which can be substituted to produce

$$
S=n_{x} l k_{B} \sqrt{\frac{\ln (2)}{\pi}}
$$

\subsubsection{Free Energy}

The free energy of the system can be calculated as a function of temperature, $k_{B} T$, and mixing FWHM, $l$, by combining equations 10,12 , and 14 to produce

$$
F=\frac{4 n_{x} l}{\sqrt{\pi \ln (2)}}(\sqrt{2}-1) J-n_{x} l k_{B} T \sqrt{\frac{\ln (2)}{\pi}} .
$$

To find the critical temperature, we minimize $F$ by setting the derivative with respect to the mixing width equal to zero and get 
LLNL-TR-497289: Simulating the Rayleigh-Taylor instability with the Ising model

$$
k_{B} T_{c}=2(\sqrt{2}-1)\left(\frac{2}{\ln (2)}\right) J
$$

This evaluates to $2.390335 \ldots J$, which differs by only $5 \%$ from the exact critical temperature for an infinite two dimensional Cartesian lattice of $\frac{2}{\ln (1+\sqrt{2})}=2.269185 \ldots J$. Our approximation is high because it slightly overestimates the mixing energy due to the second assumption. Since it is energetically favorable for spins to clump together, it is more likely for similar spins to be adjacent.

\section{Physical Laws}

By analyzing the flipping mechanism closely, we can determine the laws of motion for the system. Since spins do not maintain an intrinsic velocity vector, velocity is an instantaneous quantity. We use the conventional definition of velocity as the time rate of change in position. The concept of energy in equations 1 and 3, so we can define force as

$$
\vec{F} \equiv-\vec{\nabla} E
$$

Since energy is defined over the lattice, the gradient in our definition becomes discrete and our definition reduces to $\vec{F}=-\left(\frac{\Delta E_{u}}{\Delta h} \hat{u}+\frac{\Delta E_{d}}{\Delta h} \hat{d}+\frac{\Delta E_{r}}{\Delta h} \hat{r}+\frac{\Delta E_{l}}{\Delta h} \hat{l}\right)$, where $\hat{u}$ is the unit vector upwards, $\Delta E_{u}$ is the energy difference resulting from an upwards swap, and $\Delta h$ is the lattice spacing (see figure 7 ). We can nondimensionalize our units to the lattice spacing to get:

$$
\vec{F}=-\left(\Delta E_{u} \hat{u}+\Delta E_{d} \hat{d}+\Delta E_{r} \hat{r}+\Delta E_{l} \hat{l}\right)
$$

Here force is conceived as a vector with four elements instead of two as would be expected physically.

To relate force to spin motion we note that, since our swapping algorithm is probabilistic, our velocity will be expressed as a probability distribution. From inspection of the Metropolis algorithm with Kawasaki dynamics, the probability of a spin flipping in a direction in a single time step is equal to 
LLNL-TR-497289: Simulating the Rayleigh-Taylor instability with the Ising model

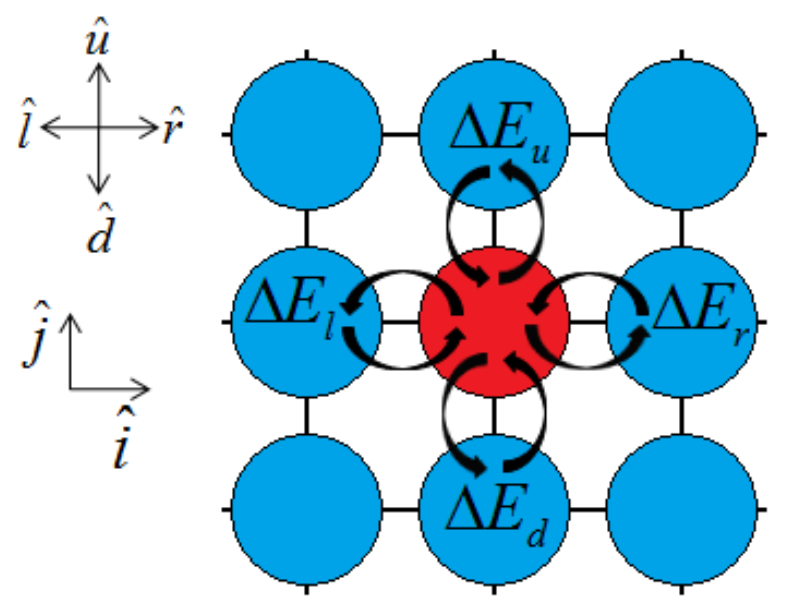

Figure 7: Unit vector and energy difference definitions

the probability that the pair is selected times the probability that it swaps. The likelihood of a pair being selected is $\frac{1}{N_{b o n d s}}$ and, given an applied force $F$, the likelihood of a selected pair swapping is $f(F)=\left\{\begin{array}{ll}1 & F<0 \\ e^{\frac{-F}{k_{B} T}} & \text { else }\end{array}\right.$. This leads to the velocity distribution

$$
\vec{v}= \begin{cases}+1 \hat{u} & \frac{f\left(F_{u}\right)}{N_{b o n d s}} \\ +1 \hat{d} & \frac{f\left(F_{d}\right)}{N_{b o n d s}} \\ +1 \hat{r} & \frac{f\left(F_{r}\right)}{N_{b o n d s}} \\ +1 \hat{l} & \frac{f\left(F_{l}\right)}{N_{\text {bonds }}} \\ 0 & 1-\frac{f\left(F_{u}\right)+f\left(F_{d}\right)+f\left(F_{r}\right)+f\left(F_{l}\right)}{N_{\text {bonds }}}\end{cases}
$$

in units of $\frac{\Delta h}{\text { time step }}$. By averaging the velocity over a large number of trials we can remove the stochastic nature of the system, convert to the conventional two element vector, and get

$$
\langle\vec{v}\rangle=\frac{f\left(F_{r}\right)-f\left(F_{l}\right)}{N_{\text {bonds }}} \hat{i}+\frac{f\left(F_{u}\right)-f\left(F_{d}\right)}{N_{\text {bonds }}} \hat{j} .
$$

Since the maximum of $f(F)$ is 1 and the minimum is 0 there will be a maximum average speed of $|\langle\vec{v}\rangle|_{\text {max }}=\frac{\sqrt{2}}{N_{b o n d s}}$. A speed limit is a consequence 
LLNL-TR-497289: Simulating the Rayleigh-Taylor instability with the Ising model

of any system possessing discrete space, discrete time, and the restriction to nearest neighbor swaps. We can modify Newton's Laws, to predict the time-averaged evolution of our system as follows:

1. The velocity average position of a body remains constant unless the body is acted upon by an external force

2. $\vec{F}^{n e t}=m \frac{d \vec{v}}{d t} \rightarrow\langle\overrightarrow{\mathbf{v}}\rangle=\frac{\mathbf{f}\left(\mathbf{F}_{\mathbf{r}}^{\text {net }}\right)-\mathbf{f}\left(\mathbf{F}_{1}^{\text {net }}\right)}{\mathbf{N}_{\text {bonds }}} \hat{\mathbf{i}}+\frac{\mathbf{f}\left(\mathbf{F}_{\mathbf{u}}^{\text {net }}\right)-\mathbf{f}\left(\mathbf{F}_{\mathbf{d}}^{\text {net }}\right)}{\mathbf{N}_{\text {bonds }}} \hat{\mathbf{j}}$

3. The mutual forces of action and reaction between two bodies are equal, opposite, and collinear

\section{$4 \quad$ Stability Conditions}

We can apply these laws to determine the stability of perturbations to the Rayleigh-Taylor instability by balancing bond and gravitational forces on the fluid interface.

\subsection{Square Wave Theory}

For a flat interface initially at $y_{0}$, a square wave perturbation is of the form $g(x)=\left\{\begin{array}{ll}y_{0}+A & 0 \leq x \leq \frac{\lambda}{2} \\ y_{0}-A & \frac{\lambda}{2} \leq x \leq \lambda\end{array}\right.$. Visually, we can determine the arc length to be $l=4 A+\lambda$ and use it to find the bond energy of a single wavelength, $E_{\text {bond }}^{\lambda}$. All $2 \lambda n_{y}-\lambda$ bonds are $-1 J$, except those on the interface, which are $+1 J$, giving an overall bond energy of

$$
\begin{aligned}
E_{b o n d} & =-\left(2 \lambda n_{y}-\lambda\right) J+2 J * l \\
& =\left(-2 n_{y}+3\right) \lambda J+8 A J .
\end{aligned}
$$

Applying equation 17, the bond force is

$$
F_{\text {bond }}=-\frac{d E_{\text {bond }}}{d A}=-8 J .
$$

The total gravitational energy with the perturbation is just the initial gravitational energy plus the energy of swapping the two blocks of spins to 
LLNL-TR-497289: Simulating the Rayleigh-Taylor instability with the Ising model

create the perturbation. $E_{\text {drop }}$ represents the energy gained by swapping a +1 spin with a vertically adjacent -1 spin. Therefore, the gravitational energy of a group of heavy spins over light spins is the product of the difference between average height of the heavy and light spins, the number of spins that swap, and $E_{\text {drop. }}$. This leads to an overall gravitational energy of

$$
\begin{aligned}
E_{\text {grav }} & =E_{\text {grav }}^{\text {flat }}-(A)\left(\frac{\lambda A}{2}\right) E_{\text {drop }} \\
& =E_{\text {grav }}^{\text {flat }}-\frac{\lambda A^{2}}{2} E_{\text {drop }},
\end{aligned}
$$

where $E_{\text {grav }}^{\text {flat }}$ is the total gravitational energy of the lattice with no perturbation to the flat interface.

Applying equation 17, the gravitational force is:

$$
F_{\text {grav }}=-\frac{d E_{\text {grav }}}{d A}=\lambda A E_{\text {drop }} .
$$

We can write the net force on the perturbation as $F^{\text {net }}=F_{\text {bond }}+F_{\text {grav }}$ and we know that, for instability growth, it must be positive. This gives

$$
E_{\text {drop }}>\frac{8}{\lambda A}
$$

as the instability condition for the square wave.

We remark that, for an interface normal to the $\hat{y}$, the lattice supports a maximum wavelength of $n_{x}$. Also, if gravity is strengthened sufficiently, any wavelength is unstable and, as the perturbation grows, it will become more energetically favorable. Lastly, if the interface between two species is initially flat, thermal effects are entirely responsible for seeding the perturbation. The process of seeding perturbations with thermal effects is stochastic. Theoretically, any perturbation will be formed, but on a timescale dependent on its wavelength and amplitude. We theorize that the wavelengths that are seen in simulations are those, permitted by equation 25 , that can be thermally formed on the shortest timescales.

\subsection{Sine Wave Theory}

The same analysis can be repeated for a smooth sine wave of the form $g(x)=$ $y_{0}+A \cdot \sin \left(\frac{2 \pi}{\lambda} x\right)$ giving a stability condition of 
LLNL-TR-497289: Simulating the Rayleigh-Taylor instability with the Ising model

$$
\begin{aligned}
E_{\text {drop }}> & \left(\frac{4}{\lambda A}\right) \sqrt{\frac{\lambda^{2}+4 \pi^{2} A^{2}}{4 \pi^{2} A^{2}}} \\
& \left(E\left(2 \pi \mid \frac{4 \pi^{2} A^{2}}{\lambda^{2}+4 \pi^{2} A^{2}}\right)-\left(\frac{\lambda^{2}}{\lambda^{2}+4 \pi^{2} A^{2}}\right) F\left(2 \pi \mid \frac{4 \pi^{2} A^{2}}{\lambda^{2}+4 \pi^{2} A^{2}}\right)\right),
\end{aligned}
$$

where $F(\phi \mid k)=\int_{0}^{\phi} \frac{d \theta}{\sqrt{1-k \sin ^{2}(\theta)}}$ and $E(\phi \mid k)=\int_{0}^{\phi} \sqrt{1-k \sin ^{2}(\theta)} d \theta$ are the incomplete elliptic integral of the first and second kind. This, however, is for a smooth sine wave where the simulation lattice can only approximate a curved perturbation with vertical and horizontal lines. Repeating the analysis once again for a discrete-space sine wave, $g(x)=y_{0}+\left[A \cdot \sin \left(\frac{2 \pi}{\lambda} x\right)\right]$, gives a stability condition of

$$
E_{\text {drop }}>\frac{16}{\lambda A}
$$

showing the sine wave is always less energetically favorable than the square wave.

\subsection{Validity of Stability Theory}

In order to apply this analysis only to the appropriate situations, we would like to determine the range of input parameters that support the assumptions of the derivations. Because Kawasaki dynamics conserves mass, our equations for $E_{\text {grav }}$ are true with any conditions. However, our equations for $E_{\text {bond }}$ in equation 21 assumes the perturbation interface is sharp, which is only an approximation for non-zero temperatures. With increasing temperature the interface becomes fuzzier and the bond energy per unit interface length increases. To formalize this effect we introduce a factor, $c_{t h}$, that represents departure from sharpness. To determine the formula for $c_{t h}$, we image a flat interface and translate the concept of bond energy from $E_{\text {bond }}\left(\hat{s}_{i}, \hat{s}_{j}\right)=\left\{\begin{array}{ll}-1 & \hat{s}_{i}=\hat{s}_{j} \\ +1 & \hat{s}_{i} \neq \hat{s}_{j}\end{array}\right.$ to $E_{\text {bond }}\left(\hat{s}_{i}, \hat{s}_{j}\right)=\left\{\begin{array}{ll}0 & \hat{s}_{i}=\hat{s}_{j} \\ +2 & \hat{s}_{i} \neq \hat{s}_{j}\end{array}\right.$, adding $N_{\text {bonds }}$ to the total bond energy. In this new system the total lattice bond energy is solely the interface energy. In the absence of gravity, we can apply equation 9 to get the energy of the lattice, apply the bond energy translation, and 
LLNL-TR-497289: Simulating the Rayleigh-Taylor instability with the Ising model

compare it with the interface energy to get

$$
\begin{aligned}
2 \cdot c_{t h} \cdot n_{x} & =n_{x} n_{y}\left\langle E_{\text {bond }}\right\rangle\left(n_{y}, k_{B} T\right)+\left(2 n_{x} n_{y}-n_{x}\right) \\
c_{t h} & =\frac{1}{2}\left(n_{y}\left(\left\langle E_{\text {bond }}\right\rangle\left(n_{y}, k_{B} T\right)+2\right)-1\right) .
\end{aligned}
$$

At low temperatures $c_{t h} \approx 1.0$, meaning our stability conditions are valid, but at high temperatures $c_{t h} \gg 1.0$ and the analysis may no longer apply. It should be noted that equation 9 is only accurate for the equilibrium bond energy. This is conservative because, if the initial interface is sharp, the interface will be fuzzier at equilibrium then at any other time.

\subsection{Results}

To test the square wave stability condition, we seeded a single wavelength on the interface of a $\lambda \times 400$ lattice with $k_{B} T=0.75$ (corresponds to $c_{t h}=$ 1.04). If the perturbation amplitude increased by 2.5 spins the simulation was recorded as growing. If the perturbation amplitude decreased by 2.5 spins the simulation was recorded as receding. Amplitude was measured at a given time step by first dividing the lattice in half vertically. Zone 1 was $0 \leq x<\frac{\lambda}{2}$ and zone 2 was $\frac{\lambda}{2} \leq x<\lambda$. Then, moving outwards (up in zone 1 and down in zone 2) from the initial interface, the majority occupant of each half row was noted. The position of the first change in the majority occupant from one row to the next determined the amplitude of each side. The overall amplitude was just the average value of the amplitudes of both zones. Frequently, especially in simulations with smaller wavelengths, lateral protrusions would separate the instability growth from the main body of spins before growth or recession could be established. This is outside the constraints of the theory, so these cases were discarded.

The results of the study appear in figures 8,9 , and $10 . E_{c r i t} \equiv \frac{8}{\lambda A}$, so stability theory predicts a unit step function at $E_{\text {drop }}-E_{\text {crit }}=0$ in each figure. The width of the transition region is caused by thermal effects on the interface length. The width does not follow simply from equation 29 because the timescale of the simulation is comparable or less than the time scale for the interface to reach thermal equilibrium.

Simulation shows that, if thermal fluctuations are used to seed the instability, only perturbations similar to square waves are seen, not a sine waves. If a sine wave is seeded on the interface, it first morphs into a square wave and then follows square wave stability theory. 
LLNL-TR-497289: Simulating the Rayleigh-Taylor instability with the Ising model

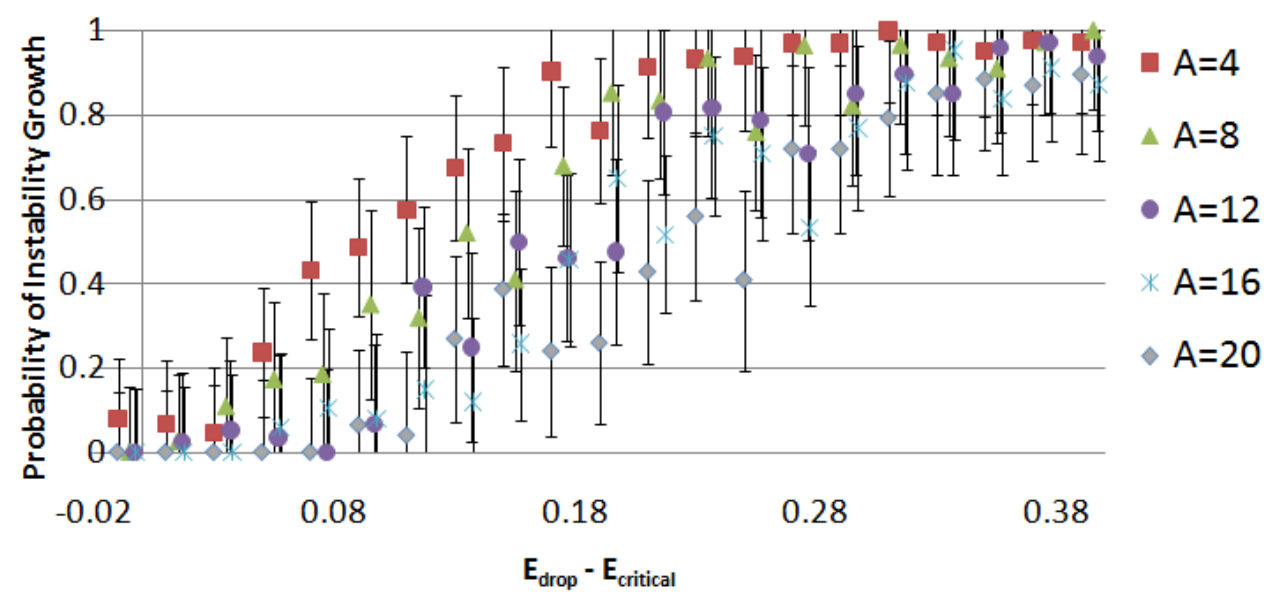

Figure 8: Stability results for $\lambda=8$ simulations

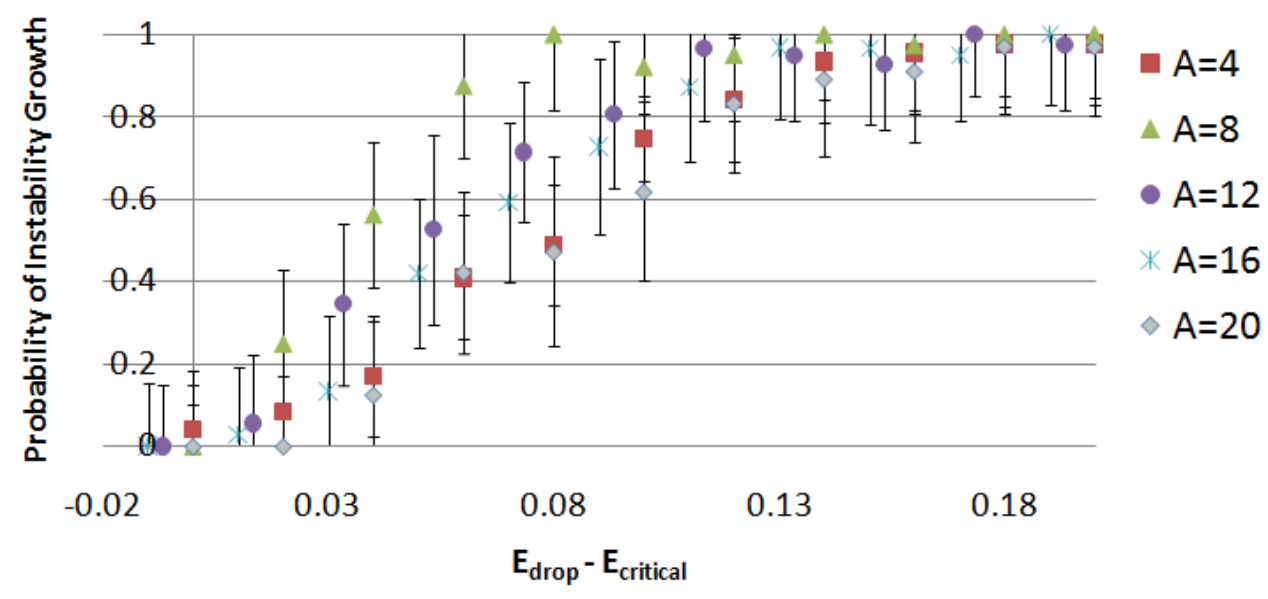

Figure 9: Stability results for $\lambda=10$ simulations 
LLNL-TR-497289: Simulating the Rayleigh-Taylor instability with the Ising model

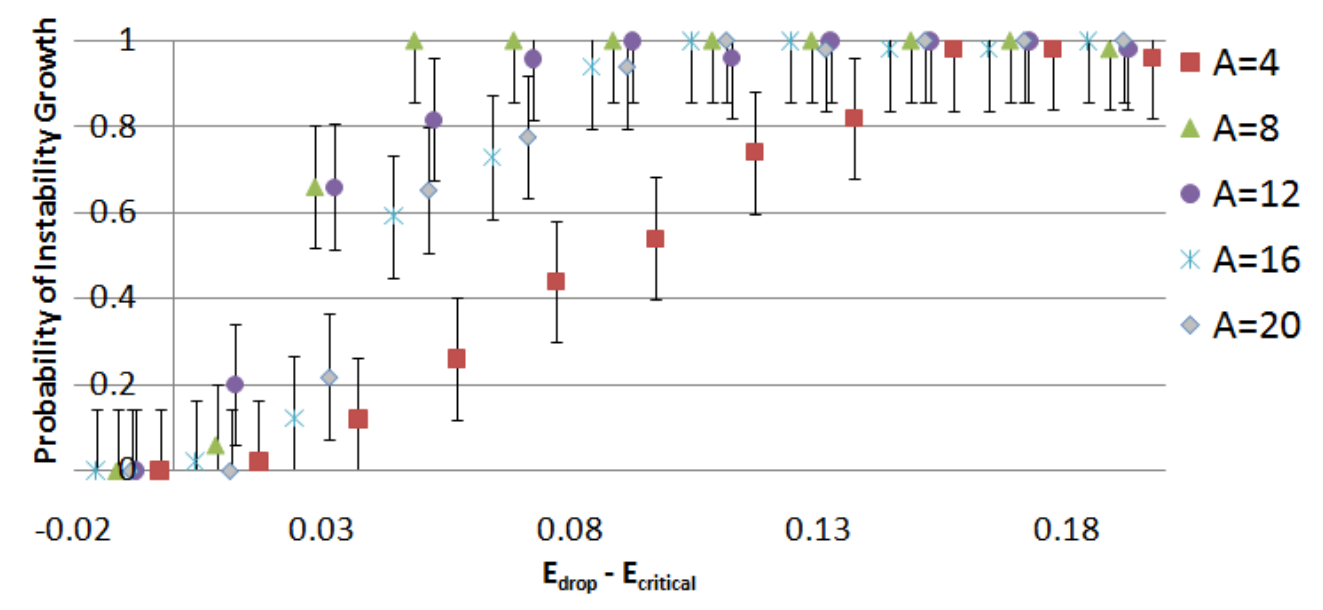

Figure 10: Stability results for $\lambda=14$ simulations

\section{$5 \quad$ Instability growth rate}

The scaling of the instability growth rate can be determined by applying our modified version of Newton's second law with the net force on interface (see equations 22 and 24). In our constrained system Newton's second law is given by $\langle\vec{v}\rangle=\frac{f\left(F_{A}\right)-f\left(F_{-A}\right)}{N_{\text {bonds }}} \hat{A}$. Since the net force felt by the interface, $\vec{F}=\left(\lambda A E_{d r o p}-8\right) \hat{A}$, is continuous, $F_{A}=-F_{-A}$, leading to

$$
\langle v\rangle=\left\langle\frac{d A}{d t}\right\rangle=c_{\text {transit }}\left(1-e^{\frac{8-\lambda A E_{\text {drop }}}{k_{B} T}}\right) .
$$

$c_{\text {transit }}$ is a factor that arises from the fact that we are swapping an entire row of spins across an extended distance on the lattice. This is a series of events that cannot be accomplished in one time step, like swapping one spin with its nearest neighbor. We know little about $c_{\text {transit }}$, so to be completely general we assume it is a function of all simulation parameters, $c_{\text {transit }}\left(A, \lambda, E_{\text {drop }}, k_{B} T, n_{y}\right)$.

We will now approximate $c_{\text {transit }}$ as independent of amplitude $\left(c_{\text {transit }}\left(A, \lambda, E_{\text {drop }}, k_{B} T, n_{y}\right) \approx\right.$ $\left.c_{\text {transit }}\left(\lambda, E_{\text {drop }}, k_{B} T, n_{y}\right)\right)$. This makes sense because, to grow the instability, we must exchange the two rows circled in figure 5 . In order to swap, these spins can either proceed along the side of the instability, through the opposing instability, or a combination of these two routes. But, as soon as they leave 
LLNL-TR-497289: Simulating the Rayleigh-Taylor instability with the Ising model

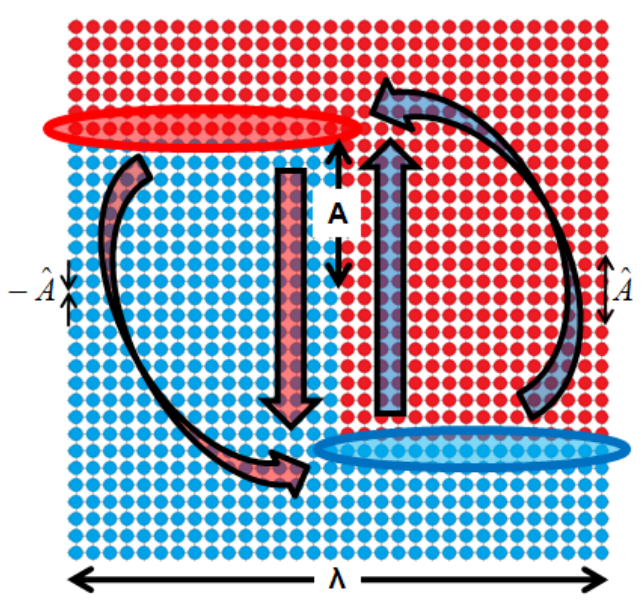

Figure 11: Seeded square wave perturbation with coordinate systems

their initial row, bond energy ceases to have an effect. Swapping along the side of the perturbation or through opposing spins entails no change in bond energy. Once they leave their home row, gravity forces them towards their destination and bond forces have little effect. On the other hand, the initial swap, freeing them from their home row, is improbable. Initially, the pair is surrounded by similar neighbors and after the swap they are surrounded by dissimilar neighbors. The leads to the proposition that the limiting factor in instability growth is the occurrence of improbable microscopic 'triggers,' not migration distance.

Confirmation of this is given in figure 5 . We can see that varying the amplitude by a factor of five causes a relatively minor spread in velocity, compared with doubling the wavelength or $E_{\text {drop }}$.

We numerically integrate equation 30 with a leapfrog method and use $c_{\text {transit }}$ as a fitting parameter to compare with simulation (see figure 5 ). However, since low temperatures (less than $\sim 1.5$ ) are necessary to simulate anything structured, the numerical integration is nearly linear. This is because $\frac{8-\lambda A E_{d r o p}}{k_{B} T} \rightarrow-\infty$ when $k_{B} T$ is small, so equation 30 becomes $\left\langle\frac{d A}{d t}\right\rangle=c_{\text {transit }}$. If the input parameters are chosen such that $8-\lambda A E_{d r o p} \approx 0$, the numerical integration will initially depart from linearity, but, as $A$ increases, the average velocity quickly approaches a constant. 
LLNL-TR-497289: Simulating the Rayleigh-Taylor instability with the Ising model

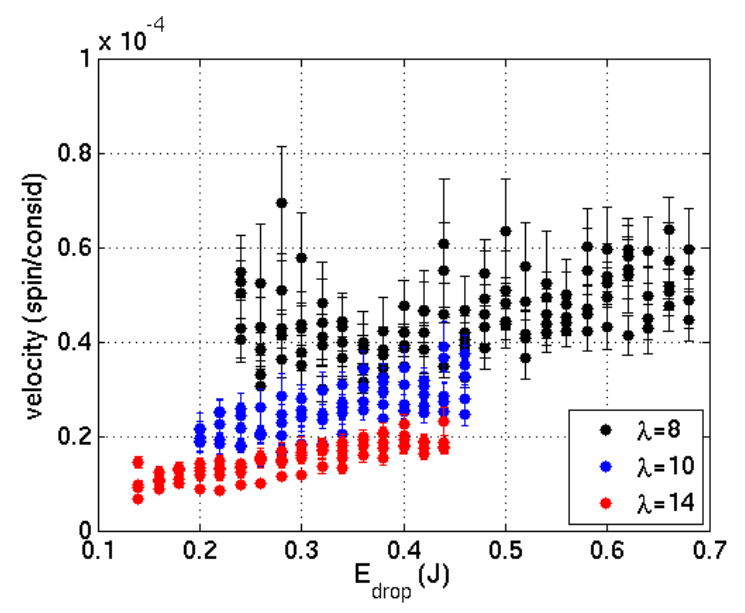

Figure 12: Velocity as a function of $E_{d r o p}, \lambda$, and $A \in\{4,8,12,16,20\}\left(k_{B} T=\right.$ $0.75 J$ and $\left.n_{y}=400\right)$

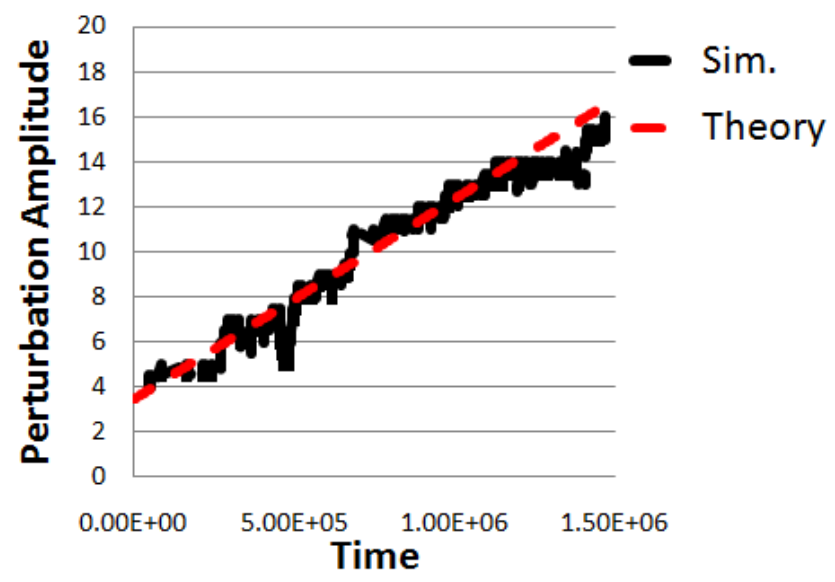

Figure 13: Comparison of growth rate theory and simulation for a square wave with initial an amplitude of four $\left(\lambda=10, E_{\text {drop }}=0.4 \mathrm{~J}, k_{B} T=0.75 \mathrm{~J}\right.$, and $\left.n_{y}=400\right)$ 
LLNL-TR-497289: Simulating the Rayleigh-Taylor instability with the Ising model

\section{Conclusions}

The Ising model, implemented with the Metropolis algorithm and Kawasaki dynamics, makes a system with its own physics, distinct from the real world. These physics are sophisticated enough to model behavior similar to the Rayleigh-Taylor instability and by better understanding these physics, we can learn how to modify the system to better reflect reality. For example, we could add a $v_{x}$ and a $v_{y}$ to each spin and modify the exchange rules to incorporate them, possibly using two body scattering laws to construct a more realistic system.

\section{Acknowledgements}

This work was done as part of LLNL's 2011 WCI Summer Student program.

LLNL-TR-497289. This work performed under the auspices of the U.S. Department of Energy by Lawrence Livermore National Laboratory under Contract DE-AC52-07NA27344. 